\title{
Evolutionary conservation of transcription factors affecting longevity.
}

Guillermo Martínez Corrales and Nazif Alic*

University College London, Institute of Healthy Ageing and GEE, Darwin Building, Gower St, WC1E 6BT, UK.

*Correspondence: n.alic@ucl.ac.uk (N.Alic)

\section{Keywords}

Transcription factors, ageing, evolution, transcriptional regulation.

\begin{abstract}
The increasing number of older people is resulting in an increased prevalence of agerelated diseases. Research has shown that the ageing process itself is a potential point of intervention. Indeed, gene expression can be optimised for health in older ages through manipulation of transcription factor (TF) activity. This review is focused on the ever-growing number of TFs whose effects on ageing are evolutionarily conserved. These regulate a plethora of functions, including stress resistance, metabolism and growth. They are engaged in complex interactions within and between different cell types, impacting the physiology of the entire organism. Since ageing is not programmed, the conservation of their effects on lifespan is most likely a reflection of the conservation of their functions in youth.
\end{abstract}




\section{Transcriptional programmes for longevity}

Most but not all animals age [1]. At a population level, ageing is universally observed as an increased likelihood of death with advancing age. In an individual animal, it manifests in a plethora of age-related changes: a decline in biological function, alterations in physiological parameters, loss of homeostatic resilience, overt disease and, ultimately, death [2]. Historically, it was often thought that animals rarely age in the wild, due to high levels of extrinsic hazards, such as predation, infection or other environmental factors, killing them before old age $[3,4]$. However, this view has recently been challenged, and in fact, ageing can be observed in several wild populations of many different animal species [5-7] . Specifically, in human populations, the importance of ageing has significantly risen at a global scale. Indeed, chronic diseases have increased their significance in the last decades not only in developed countries, but also in most developing countries [8,9]. With profound changes to human societies, including the development of modern medicine, human life expectancy has increased dramatically and so has the proportion of older people [10]. As a result, humans now face new health challenges; age-related diseases previously almost unknown are reaching epidemic proportions.

The appearance of ageing as a stochastic and intractable process is in stark contrast with the plasticity of lifespan observed in studies of animal model organisms. Already three decades ago, single-gene mutants in $C$. elegans were identified that had a much longer lifespan than the wild-type [11, 12]. This came as quite a surprise because previous work in population genetics and evolution showed that ageing is a highly polygenic trait, unlikely to be under control of single genes [13, 14]. It was even more surprising that the effects of these genes on ageing were evolutionarily conserved [1518]. Subsequent studies have elucidated an ever-increasing number of genetic, environmental and pharmacological interventions that can extend healthy lifespan. They all appear to act, at least in part, by regulating gene expression to promote health and longevity. Indeed, one of the first pro-longevity genes encoded a transcription factor (TF), the Caenorhabditis elegans daf-16.

TFs recognise and bind specific DNA sequences in the genome to regulate transcription of their target genes. The pro-longevity effects of DAF-16 were soon ascribed to its ability to orchestrate genome-wide changes in gene expression where 
the impact of each DAF-16 target gene summed up to produce a substantial effect on lifespan [19, 20]. DAF-16 is not alone. The list of TFs whose activity can be manipulated to extend lifespan and whose effects are evolutionarily conserved is evergrowing. But why are there so many? What genes and processes do they all regulate? Why are their effects on lifespan conserved between such different animal species? In this review, we will propose answers to these questions, focusing on recent developments in the field.

In particular, we will focus on TFs whose activity can be altered to extend lifespan, and not simply shorten it, because they reveal what limits the longevity in the wild-type animal. Some of them promote longevity, whereas others promote ageing and longevity occurs due to their inhibition. Specifically, we will focus on the TFs where the TF family or direct orthologues have been demonstrated to play a role in ageing in at least two different species. These are summarised in Table 1.

\section{Stress less}

Studying the transcriptional programmes that promote longevity represents an opportunity to understand ageing and to harness this knowledge for the design of interventions that maintain health in older humans. An extensively studied and wellconserved family of TFs implicated in ageing is the Fork Head family. DAF-16 is a Fork Head TF of the box O subfamily (FOXO). It acts downstream of insulin/insulin-like growth factor signalling (IIS) and it is required for IIS inhibition to extend lifespan in worms and flies [21]. The relevance of FOXOs to ageing has been confirmed in numerous animal models, including the immortal hydra, worms, or flies [17, 18, 2225]. FOXOs might also be relevant in humans where variants of FOXO3 have been associated with longevity in several studies, including a recent meta-analysis [26, 27]. However, some studies were not able to replicate these findings [28]. In addition, at least one more forkhead TF, FoxA, can promote longevity, with FoxA orthologues extending lifespan in both worms and flies [29, 30].

Transcriptional programmes triggered by FOXOs have highlighted several processes that promote longevity. These include several stress-responsive effector pathways, such as autophagy, protein refolding and turnover, anti-oxidant and xenobiotic defences [23, 31-34]. Highlighting their importance, similar processes can be activated by other longevity-promoting TFs. These include nuclear factor erythroid 2-related 
factor 2(NRF2) orthologues, which are known to be involved in xenobiotic detoxification [35-38], as well as the endoplasmic reticulum unfolded protein response mediator, X-box binding protein 1 (XBP1) [39, 40]. This consistent presence of pathways that limit or repair molecular damage in transcriptional programmes that promote longevity has reinforced the view that ageing results from accumulation of age-related molecular damage.

In addition to limiting molecular and cellular damage, FOXOs (and FOXAs) also have a well-established and important role to play in metabolic homeostasis $[21,23,29,33$, 41] (reviewed in [42]). This, in turn, implicates metabolism and its control as an important factor in longevity. Indeed, a vast number of lifespan-extending interventions influence general metabolism and numerous other longevity-relevant TFs, such as those of the ETS family or MYC, are implicated in the regulation of cellular and organismal metabolism [33, 43-46]. For example, mice haploinsufficient for MYC live longer than wild-type and show improvements in metabolic function but not an increase in xenobiotic resistance, suggesting that at least some forms of stress resistance can be uncoupled from longevity [44]. Even TFs clearly responsive to stress, such as XBP1 [47] are now known to contribute to longevity via metabolic control in worms [48] and a recent study in mice demonstrated that XBP1 knock-out mutants show an accelerated decay in the metabolic status and function of their retina [49]. Clearly, metabolism is emerging as a key longevity determinant.

However, the exact recipe for a metabolic switch that promotes healthy lifespan is not fully understood. Historically, lifespan extension treatments like restricting nutrient intake without malnutrition, or downregulation of IIS, correlated with a general reduction of metabolic rate and glycolysis, and a delay in mitochondrial functional failure [50-52]. However, the universality of this conclusion has been challenged [53], and, in fact, glycolysis needs to be maintained to promote healthy ageing in Drosophila males [54]. The metabolic shift that drives longevity might be substantially contextdependent. Indeed, enhanced activity of FOXO in the muscle can reduce circulating sugars [23], whereas the activity of the pro-longevity ETS TF, Aop, in the fat body appears to have opposite outcome [34]. Additionally, recent work has highlighted a vital role played by lipid metabolism in transcriptional regulation of longevity, [32, 33 , $48,55,56$ ] with a complex picture emerging from a number of studies [57]. Although this metabolic reprogramming by longevity-relevant TFs is extensive and varied, it is 
also clearly important for lifespan and it might act in parallel or be interlined with their roles in limiting molecular damage.

TFs that regulate growth and proliferation can also influence lifespan. This includes MYC, a TF extensively studied for its role in promoting growth and proliferation in the context of development and cancer (reviewed in [58]). Interestingly, MYC can activate transcription by all three nuclear RNA polymerases to promote ribosome biogenesis [59-61]. And indeed, recent findings indicate that longevity can be determined not only by RNA polymerase II, the polymerase that transcribes coding genes, but also that partially inhibiting the activity of RNA polymerase III (Pol III) can extend lifespan in worms and flies [62]. This polymerase is responsible for transcribing short, non-coding RNAs such as tRNAs and the 5S rRNA and is typically considered to have growthpromoting and not stress-related cellular function(s) [63]. Importantly, curtailing growth-promoting functions only in the adult is sufficient to extend lifespan [62], indicating longevity is not directly caused by altered development. During fly ageing, Pol III acts downstream of Target of rapamycin kinase complex 1 (TORC1). TORC1 delineates a growth-promoting pathway that is a crucial determinant of lifespan in numerous animal species [64]. The importance of TORC1 in ageing has stimulated research into the links between functions that promote growth and longevity. Accumulating evidence shows that inhibiting functions associated with growth (e.g. ribosome biogenesis and translation) is sufficient to extend lifespan, but the mechanisms are not fully understood [65]. Still, it is clear that in addition to counteracting molecular damage and rebalancing metabolism, transcriptional inhibition of growth promoting processes can also extend lifespan.

Several recent studies have highlighted that ageing of cells, tissues and organs is accompanied by changes in their transcriptomes [32, 66-68]. It is tempting to speculate that modulating the activity of lifespan-relevant TFs allows cells to maintain a "youthful" transcriptome for longer. Indeed, many of the longevity-promoting processes, such as autophagy, protein refolding and turnover, or mitochondrial activity decrease in aged animals in multiple species, including worms [69], flies [23, 54, 70] and mammals including humans [71]. Metabolism is profoundly altered with age in a great variety of animals [42]. For example, a recent study in Drosophila males showed that transcription of glycolytic enzymes declines with age [54]. At the same time, some of the growth-promoting processes show a relative increase or misregulation with age 
[67]. Therefore, the manipulation of specific TFs might reinforce the control of these processes, preventing their age-induced "drift". In addition, there might be an agerelated alteration in the activity of ageing-relevant TFs themselves. A recent report analysed the DNA binding pattern of FOXO in Drosophila and found that FOXO's presence on the DNA drastically decreases with age [32]. Hence, it is possible that activating FOXO might counteract ageing by preventing its own function from declining.

Altogether, the ongoing work on transcriptional programmes orchestrated by different TFs to promote longevity hasn't highlighted a single, central cellular and organismal process. Instead, it has uncovered a panel of interconnected functions, some of which promote stress resistance whereas others alter metabolic and growth-promoting processes. It is likely that modulating all of them enhances health and survival in older age. It will be interesting to understand if their manipulations act to correct age-related transcriptional changes and to what extent. This would imply that ageing could be in part caused by a loss of transcriptional control of gene expression.

\section{Friends and foes}

Ageing manifests at a number of levels, from molecular to organismal, and affects a broad range of processes. Several manifestations are consistently observed between and within animal species and have been called the "hallmarks" of ageing [72]. Therefore, it is not surprising that an ever-increasing number of TFs can impact ageing, as each TF might be targeting one of the specific process limiting lifespan. However, this is not what is observed. Looking at specific TFs reveals that they each have a broad range of targets, and for example, FOXO affects stress resistance genes, metabolism and inhibits growth-related functions [73]. So why are we finding so many longevity-related TFs? We believe the answer might lie in the complexity of interactions that regulate animal physiology.

TFs do not act alone but engage in complex regulatory networks where they modulate each other's activity and outputs. These complex interactions, which define gene regulatory networks, have been extensively studied during animal development in the last 50 years $[74,75]$. They are also likely to be relevant in the context of ageing. For example, FOXOs have been observed to antagonise MYC or to oppose its transcriptional output in a number of contexts $[76,77]$. It is hence conceivable that 
activating FOXO and reducing the activity of MYC could trigger a similar transcriptional programme that leads to an extension of animal lifespan. Elaborating this idea, it becomes likely that the complex circuitry formed by interactions between a number of TFs within a cell can be altered and fine-tuned by multiple individual TFs to achieve the same or similar longevity programme. Findings consistent with this idea have been documented for FOXO and two ETS factors, Aop and Pnt, in fruit flies. These three TFs appear to modulate the transcriptional outcome of each other and converge to regulate similar sets of genes [33]. Importantly, longevity can be achieved by direct manipulation of any one of the three. The relevant network might be even bigger as FOXOs interact with a number of other TFs, in a number of ways. In C. elegans, the examples include HSF-1, SKN-1 (the worm Nrf-2 orthologue), GATA factors and PQM1 , all of which are relevant to lifespan in this organism [55, 78, 79]. Hence, many TFs might be triggering the same or similar transcriptional programme to promote longevity.

Multicellular organisms are composed of different cell types, each specialised for specific functions. Interestingly, changing the transcriptional programme within a single cell type can be sufficient to increase the longevity of the entire organism. The differences between cell types arise as the consequence of differences in gene expression orchestrated during development in such a way that each cell type essentially has a specific combination of TFs with certain interactions possible between them [75]. Hence, different TFs will act in different cell types to promote longevity. We will use Drosophila as an example to illustrate this point. In Drosophila, FOXO extends lifespan independently in the muscle [23] or in the fat body $[17,18]$. However, it does not appear to promote longevity solely from the gut [34], where Fkh appears to be relevant [29]. Two GATA factors, Srp and GATAe, are required in the fat body and the gut, respectively, to mediate the effects of dietary restriction on lifespan [80]. Several ETS factors impact lifespan from exclusive tissues. For example, a reduction in Eip74EF can extend lifespan from neurons, where normally it is highly expressed, but not from the gut or fat body, and Ets97D and Pnt have an effect from the fat body and not neurons [33]. The gut, and specifically the stem cell population it harbours, is also the site whence RNA polymerase III activity impacts longevity [62]. Similar tissue or cell-type specificity has been observed in the worm, with often equivalent TFs acting from equivalent tissues in different species [17, 18, 62, 81]. 
Interestingly, where a TF can act from different tissues, it triggers a transcriptional programme that is unique to each tissue $[73,82]$. Such cell-type specificity also means that a given TF can be beneficial in one tissue but detrimental in another. Increasing FOXO activity in neurons shortens lifespan [17, 33]. In Drosophila, ubiquitous, heterozygous loss of Myc promotes longevity [83], but its gain of function in enterocytes appears beneficial on high yeast diets [84]. Similarly, inhibiting Pol III in intestinal stem cells in flies promotes longevity but the same phenotype is observed upon the loss of its negative regulator, the TF Maf1, in worms and mice [85, 86], where the critical tissues for Maf1 loss-of-function might be metabolic tissues such as liver [87]. Hence, there appear to be numerous distinct longevity programmes occurring in distinct cell types. They might again converge at the level of whole animal physiology: changes in TF activity in one cell type will often trigger cell-to-cell signalling cascades that will affect TF activity in other cell types [17, 23, 39, 55, 88]. A summary of the different TFs that directly influence lifespan tissue-specifically in Drosophila is shown in Figure 1.

This complexity of cell-specific transcriptional networks, as well as the cell-type complexity present in an adult animal and the coordination of their functions, might be able to account for the ever-growing number of TFs whose manipulation appears sufficient to promote longevity. It will be interesting to understand how many truly independent ways there are to improve an animal's health and survival. Genetic screens in worms, and drug studies in the fly, converge on a number that is smaller than the number of TFs involved in longevity: a handful of pathways whose effects are additive [89-91].

\section{From the cradle to the grave}

Ageing is not thought to exist to serve a purpose and hence, is not considered to be programmed in the way development is [92]. Indeed, there is no a priori reason that two species should age in the same way and die of the same causes [1,93]. And yet, specific alterations in activities of equivalent TFs, or members of the same TF families, can extend healthy lifespan across taxa, sometimes more than doubling it [24]. This conservation is evident at the level of orthologous TFs such as DAF-16 in worms, FOXO in flies, FOXO3 in humans and even down to Fork Head TFs in yeast [94]. It can also be observed within TF paralogues in a single species, as appears to be the case for ETS or GATA factors in flies and worms [33, 43, 80]. This conservation of 
function is reflected in an apparent conservation of their transcriptional targets. For example, certain genes are consistently regulated by FOXOs in multiple species [95]. Furthermore, FOXO's interacting partners also appear conserved. An interaction between the FOXO family and ETS family is found in both Drosophila and C. elegans $[33,34,43]$. It might be present in a number of other species, as indicated by betweenspecies comparison of FOXO targets [95]. Overall, there is a strong implication that the ancestral TFs and their partners had relevance to ageing.

The evolutionary conservation of TF function per se is not so surprising. The primary DNA sequences recognised by orthologous/paralogous TFs are well conserved between Drosophila, mice and humans, where they are repeatedly used to drive similar cellular or organismal processes $[96,97]$. But it is surprising that their role in ageing is maintained between species. Since ageing is not programmed, why do we observe such evolutionary conservation? And why haven't animals evolved means to maintain the activity of these TFs at levels that maximise lifespan? The answer to both questions might lie in the multiple phenotypic effects these TFs have and the roles they play before organismal senescence takes place.

A single gene can influence multiple phenotypes. If an allele of the gene occurs that improves fitness in early life, for example promoting growth or increasing reproductive output, natural selection can favour this allele even if it increases the likelihood of dying with age. This is because of the "selection shadow": the force of natural selections weakens with age due to a decline in population size with time, as individuals in the population die from extrinsic hazards even in the absence of ageing itself. Hence, there are fewer individuals that display a later phenotype of an allele. This means that if an allele is antagonistically pleiotropic and promotes early-life fitness at the expense of later life survival, it can still be favoured by natural selection. This antagonistic pleiotropy was proposed as the reason for the existence of ageing [4, 98]. It has been recently reviewed in [14] and [92].

For some TFs and the processes they drive, it is easy to conceptualise how their activity might be antagonistically pleiotropic. For example, MYC is required for growth to reach reproductive age, so its activity will be optimised for this function even at the expense of later life health $[46,59]$. Similar could be said for Pol III and other growthpromoting transcriptional processes. For the TFs that control metabolism, their activity 
could be fine-tuned for optimal early-life metabolic state. Other TFs are involved in cellular processes that show antagonistically pleiotropic effects, such as p53 and its role in promoting cellular senescence, a process that prevents cancer and helps wound healing while driving mammalian ageing [99].

For other TFs, this antagonistic pleiotropy might not be obvious. For TFs that control stress resistance and similar processes, a trade-off is often invoked, where the distribution of resources, such as energy, needs to be balanced between processes ensuring reproductive success and those ensuring stress resistance [92]. A trade-off could be mediated by the pathways that regulate several of the relevant TFs, such as the IIS pathway or TORC1, which control key aspects of an animal's physiology promoting reproductive success while repressing stress responses [92]. IIS and TORC1 are necessary for development and organismal growth, and this is evolutionarily conserved from insects to humans (recently reviewed in [100]). In all these cases, the signalling pathways will control TF activity to maximise early fitness at the expense of health in older age. However, experimental evidence indicates that, in mechanistic terms, the trade-off between longevity and reproduction can be avoided [92]. The evolutionary explanation for why the activity of the pro-longevity TFs hasn't been optimised for longer lifespan might be even simpler: there has never been a chance or a need to do that. Extrinsic hazards killed the animals first. Indeed, given the opportunity, longer lifespan can be evolved [1] and in some species, this might occur through the TFs mentioned in this review and the genes they regulate.

So why do we find TFs whose effects on ageing are evolutionarily conserved? We propose that what has been conserved through evolution are the functions of these TFs in processes that ensure fitness. This essentially refers to processes that are relevant in the part of the animal's life history where natural selection has been able to act, in the animal's "early" life. Thus, the conserved effects of the TFs on lifespan are simply a consequence of their conserved functions in early life.

\section{Concluding remarks}

While the number of TF whose effects on ageing are evolutionarily conserved has dramatically expanded, we still do not have a complete understanding of how the gene expression programmes they regulate act to promote longevity. Characterising these 
longevity transcriptional programmes is on-going, facilitated by a number of genomewide approaches in use. However, this is likely to provide only a partial answer as it is often a reductionist approach. For a full understanding of how these TFs drive a phenotype that is a characteristic of the entire organism, such as ageing, we still need to decipher their interactions within cells, between cells and across different times in an animal's life. 


\section{Additional material}

\section{Table}

\begin{tabular}{|c|c|c|c|c|}
\hline \multirow{2}{*}{$\begin{array}{l}\text { TF or TF } \\
\text { family }\end{array}$} & \multicolumn{4}{|c|}{ Organism } \\
\hline & C. elegans & D. melanogaster & M. musculus & H. sapiens \\
\hline $\begin{array}{l}\text { Fork } \\
\text { Head }\end{array}$ & $\begin{array}{l}\text { DAF-16 [24, 101, } \\
\text { 102] } \\
\text { PHA-4 [30] }\end{array}$ & $\begin{array}{l}\text { FOXO }[17,18] \\
\text { FKH }[29]\end{array}$ & $\begin{array}{l}\text { FOXO3 } \\
\text { (mediator of CR) } \\
{[103]}\end{array}$ & $\begin{array}{l}\text { FOXO1 [104] } \\
\text { FOXO3 }[27, \\
105]\end{array}$ \\
\hline GATA & $\begin{array}{l}\text { ELT-2 }[55,106] \\
\text { ELT-3/5/6 }[107,108]\end{array}$ & $\begin{array}{l}\text { Srp [80] } \\
\text { GATAe [80] }\end{array}$ & & \\
\hline ETS & $\begin{array}{l}\text { LIN-1 [33] } \\
\text { ETS-4 [43] }\end{array}$ & $\begin{array}{l}\text { Aop [34], Pnt [33], } \\
\text { Eip74EF [33], Ets21C } \\
\text { [33], Ets97D [33] }\end{array}$ & & \\
\hline Nrf2 & SKN-1 [109] & CncC $[35,36]$ & NRF2 [110] & \\
\hline CREB & CRH-1 [111] & & CREB [112] & \\
\hline Myc & $\begin{array}{l}\text { Myc-Mondo-like } \\
\text { complexes [88] }\end{array}$ & $\mathrm{dMyc}[83,84]$ & MYC [44] & \\
\hline Maf1 & $\begin{array}{l}\text { Maf1 [85], Pol III } \\
\text { itself [62] }\end{array}$ & Maf1, Pol III itself [62] & Maf1 [86] & \\
\hline P53 & CEP-1 $[113,114]$ & p53 [115] & P53[116-118] & p53 $[119,120]$ \\
\hline Xbp1 & $\mathrm{XBP}-1[47,48]$ & Xbp1 [39] & & \\
\hline
\end{tabular}

Table 1. Evolutionary conservation of TFs involved in ageing. This table summarises the different TFs or TF families whose activity has been found to impact lifespan across taxa, including data from 4 species. TFs highlighted in green are beneficial for lifespan, the ones in red have been reported as limiting wild-type lifespan. In the case of TFs that have been reported both as lifespan-promoting and lifespan-limiting factors, they are highlighted in blue. References to the original publications are noted next to the TFs.

\section{Figure legend}

Figure 1. The Drosophila TFs act in different organs to impact longevity. Schematic diagram of the different TFs acting tissue-independently in the adult Drosophila melanogaster to regulate lifespan. Four different key tissues are shown: Skeletal muscle, brain, fat body and midgut. The TFs that promote lifespan from the specific tissue or cell-type are highlighted in green, and the ones that limit it, in red. TFs highlighted in blue indicate either that they can both length and shorten lifespan (e.g. the hormetic effect of cncC [38]), or that they appear to impact lifespan only under certain conditions (e.g. Srp and GATAe under dietary restriction [33]). Created with BioRender.com. 
Skeletal muscle

Fat body

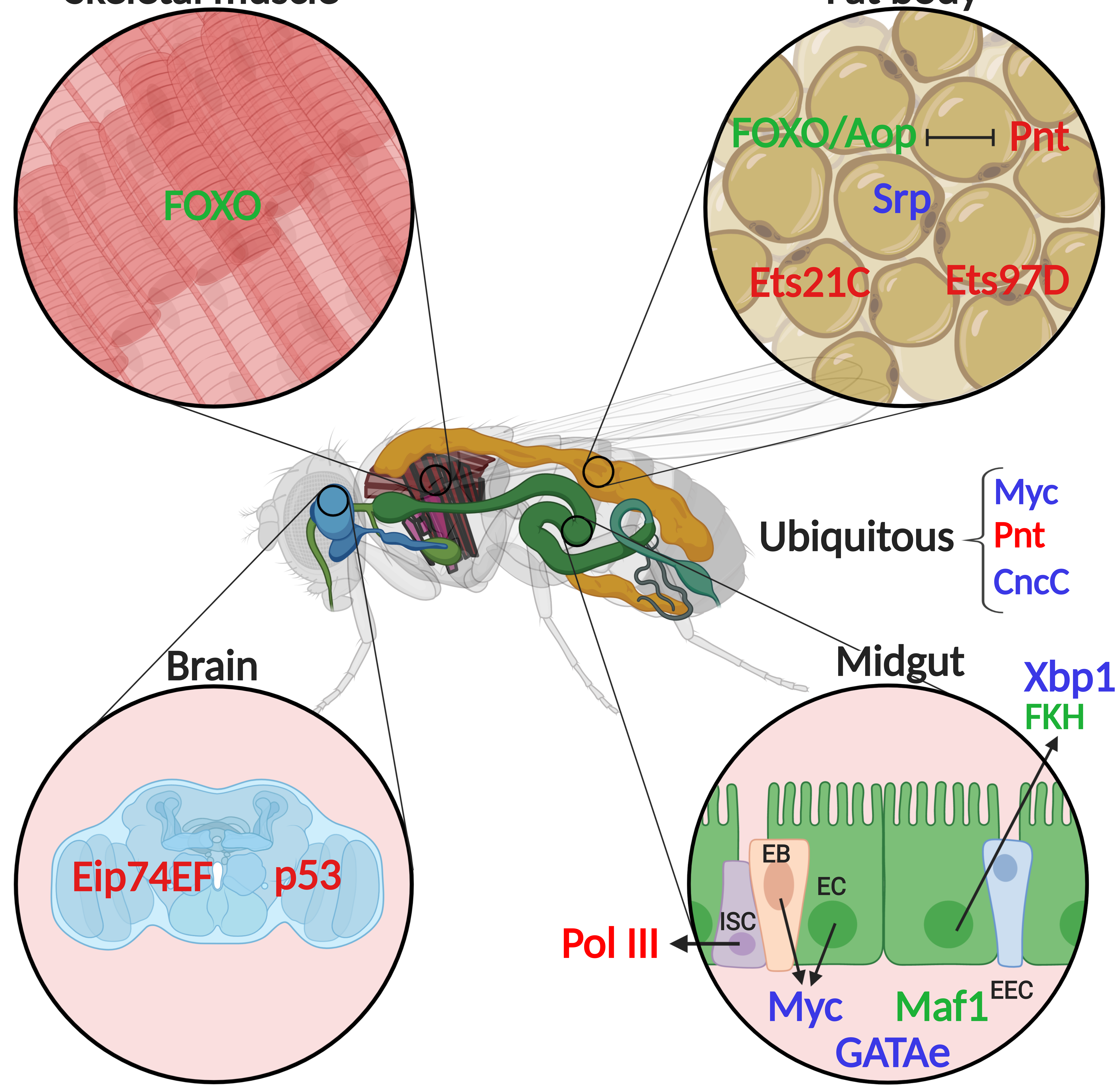




\section{Acknowledgements}

We thank the members of the Institute of Healthy Ageing for helpful discussions on the manuscript. This work was supported by the Biotechnology and Biological Sciences Research Council (BBSRC grant BB/R014507/1). We apologize to colleagues whose work could not be cited due to space limitations.

\section{Disclaimer statement}

The authors declare no competing interests related to this work.

\section{References}

1. Jones, O.R. et al. (2014) Diversity of ageing across the tree of life. Nature 505 (7482), 169.

2. Niccoli, T. and Partridge, L. (2012) Ageing as a risk factor for disease. Current biology 22 (17), R741R752.

3. Haldane, J.B.S. (1942) New paths in genetics, George allen \& Unwin.

4. Williams, G.C. (1957) Pleiotropy, natural selection, and the evolution of senescence. evolution, 398411.

5. Warner, D.A. et al. (2016) Decades of field data reveal that turtles senesce in the wild. Proceedings of the National Academy of Sciences 113 (23), 6502-6507.

6. Nussey, D.H. et al. (2013) Senescence in natural populations of animals: widespread evidence and its implications for bio-gerontology. Ageing research reviews 12 (1), 214-225.

7. Froy, H. et al. (2019) Senescence in immunity against helminth parasites predicts adult mortality in a wild mammal. Science 365 (6459), 1296-1298.

8. Metrics, I.f.H. and Evaluation, Findings from the global burden of disease study 2017, IHME Seattle, WA, 2018.

9. Yach, D. et al. (2004) The global burden of chronic diseases: overcoming impediments to prevention and control. Jama 291 (21), 2616-2622.

10. Christensen, K. et al. (2009) Ageing populations: the challenges ahead. Lancet 374 (9696), 1196208.

11. Friedman, D.B. and Johnson, T.E. (1988) A mutation in the age-1 gene in Caenorhabditis elegans lengthens life and reduces hermaphrodite fertility. Genetics 118 (1), 75-86.

12. Kenyon, C. et al. (1993) A C. elegans mutant that lives twice as long as wild type. Nature 366 (6454), 461.

13. Kenyon, C.J. (2010) The genetics of ageing. Nature 464 (7288), 504.

14. Flatt, T. and Partridge, L. (2018) Horizons in the evolution of aging. BMC biology 16 (1), 1-13.

15. Tatar, M. et al. (2001) A mutant Drosophila insulin receptor homolog that extends life-span and impairs neuroendocrine function. Science 292 (5514), 107-10.

16. Clancy, D.J. et al. (2001) Extension of life-span by loss of CHICO, a Drosophila insulin receptor substrate protein. Science 292 (5514), 104-106.

17. Hwangbo, D.S. et al. (2004) Drosophila dFOXO controls lifespan and regulates insulin signalling in brain and fat body. Nature 429 (6991), 562.

18. Giannakou, M.E. et al. (2004) Long-lived Drosophila with overexpressed dFOXO in adult fat body. Science 305 (5682), 361-361.

19. Murphy, C.T. et al. (2003) Genes that act downstream of DAF-16 to influence the lifespan of Caenorhabditis elegans. Nature 424 (6946), 277. 
20. Lee, S.S. et al. (2003) DAF-16 target genes that control C. elegans life-span and metabolism. science 300 (5619), 644-647.

21. Martins, R. et al. (2016) Long live FOXO: unraveling the role of FOXO proteins in aging and longevity. Aging cell 15 (2), 196-207.

22. Giannakou, M.E. and Partridge, L. (2007) Role of insulin-like signalling in Drosophila lifespan. Trends Biochem Sci 32 (4), 180-8.

23. Demontis, F. and Perrimon, N. (2010) FOXO/4E-BP signaling in Drosophila muscles regulates organism-wide proteostasis during aging. Cell 143 (5), 813-825.

24. Lin, K. et al. (1997) daf-16: An HNF-3/forkhead family member that can function to double the lifespan of Caenorhabditis elegans. Science 278 (5341), 1319-1322.

25. Boehm, A.-M. et al. (2012) FoxO is a critical regulator of stem cell maintenance in immortal Hydra. Proceedings of the National Academy of Sciences 109 (48), 19697-19702.

26. Timmers, P.R. et al. (2019) Genomics of 1 million parent lifespans implicates novel pathways and common diseases and distinguishes survival chances. elife 8, e39856.

27. Deelen, J. et al. (2019) A meta-analysis of genome-wide association studies identifies multiple longevity genes. Nat Commun 10 (1), 3669.

28. Pilling, L.C. et al. (2016) Human longevity is influenced by many genetic variants: evidence from 75,000 UK Biobank participants. Aging (Albany NY) 8 (3), 547.

29. Bolukbasi, E. et al. (2017) Intestinal fork head regulates nutrient absorption and promotes longevity. Cell reports 21 (3), 641-653.

30. Panowski, S.H. et al. (2007) PHA-4/Foxa mediates diet-restriction-induced longevity of C. elegans. Nature 447 (7144), 550.

31. Li, S.T. et al. (2019) DAF-16 stabilizes the aging transcriptome and is activated in mid-aged Caenorhabditis elegans to cope with internal stress. Aging cell 18 (3), e12896.

32. Birnbaum, A. et al. (2019) Age-dependent changes in transcription factor FOXO targeting in female Drosophila. Frontiers in genetics 10, 312.

33. Dobson, A.J. et al. (2019) Longevity is determined by ETS transcription factors in multiple tissues and diverse species. PLoS genetics 15 (7), e1008212.

34. Alic, N. et al. (2014) Interplay of dFOXO and two ETS-family transcription factors determines lifespan in Drosophila melanogaster. PLoS genetics 10 (9), e1004619.

35. Spiers, J.G. et al. (2019) Drosophila Nrf2/Keap1 Mediated Redox Signaling Supports Synaptic Function and Longevity and Impacts on Circadian Activity. Frontiers in Molecular Neuroscience 12.

36. Castillo-Quan, J.I. et al. (2016) Lithium promotes longevity through GSK3/NRF2-dependent hormesis. Cell reports 15 (3), 638-650.

37. Sykiotis, G.P. and Bohmann, D. (2008) Keap1/Nrf2 signaling regulates oxidative stress tolerance and lifespan in Drosophila. Developmental cell 14 (1), 76-85.

38. Tsakiri, E.N. et al. (2019) Hyperactivation of Nrf2 increases stress tolerance at the cost of aging acceleration due to metabolic deregulation. Aging cell 18 (1), e12845.

39. Luis, N.M. et al. (2016) Intestinal IRE1 is required for increased triglyceride metabolism and longer lifespan under dietary restriction. Cell reports 17 (5), 1207-1216.

40. Henis-Korenblit, S. et al. (2010) Insulin/IGF-1 signaling mutants reprogram ER stress response regulators to promote longevity. Proceedings of the National Academy of Sciences 107 (21), 97309735.

41. Wang, M.C. et al. (2008) Fat metabolism links germline stem cells and longevity in C. elegans. science 322 (5903), 957-960.

42. López-Otín, C. et al. (2016) Metabolic control of longevity. Cell 166 (4), 802-821.

43. Thyagarajan, B. et al. (2010) ETS-4 is a transcriptional regulator of life span in Caenorhabditis elegans. PLoS genetics 6 (9), e1001125.

44. Hofmann, J.W. et al. (2015) Reduced expression of MYC increases longevity and enhances healthspan. Cell 160 (3), 477-488. 
45. Slack, C. et al. (2015) The Ras-Erk-ETS-signaling pathway is a drug target for longevity. Cell 162 (1), 72-83.

46. Pierce, S.B. et al. (2004) dMyc is required for larval growth and endoreplication in Drosophila. Development 131 (10), 2317-2327.

47. Taylor, R.C. and Dillin, A. (2013) XBP-1 is a cell-nonautonomous regulator of stress resistance and longevity. Cell 153 (7), 1435-1447.

48. Imanikia, S. et al. (2019) XBP-1 remodels lipid metabolism to extend longevity. Cell reports 28 (3), 581-589. e4.

49. McLaughlin, T. et al. (2018) Loss of XBP1 accelerates age-related decline in retinal function and neurodegeneration. Molecular neurodegeneration 13 (1), 16.

50. Feuers, R.J. et al. (1989) Effect of chronic caloric restriction on hepatic enzymes of intermediary metabolism in the male Fischer 344 rat. Mechanisms of ageing and development 48 (2), 179-189.

51. Van Voorhies, W.A. and Ward, S. (1999) Genetic and environmental conditions that increase longevity in Caenorhabditis elegans decrease metabolic rate. Proceedings of the National Academy of Sciences 96 (20), 11399-11403.

52. Ruetenik, A. and Barrientos, A. (2015) Dietary restriction, mitochondrial function and aging: from yeast to humans. Biochimica et Biophysica Acta (BBA)-Bioenergetics 1847 (11), 1434-1447.

53. Hulbert, A. et al. (2004) Metabolic rate is not reduced by dietary-restriction or by lowered insulin/IGF-1 signalling and is not correlated with individual lifespan in Drosophila melanogaster. Experimental gerontology 39 (8), 1137-1143.

54. Ma, Z. et al. (2018) Epigenetic drift of H3K27me3 in aging links glycolysis to healthy longevity in Drosophila. Elife 7, e35368.

55. Zhang, P. et al. (2013) Direct and indirect gene regulation by a life-extending FOXO protein in C. elegans: roles for GATA factors and lipid gene regulators. Cell metabolism 17 (1), 85-100.

56. Han, S. et al. (2017) Mono-unsaturated fatty acids link H3K4me3 modifiers to C. elegans lifespan. Nature 544 (7649), 185.

57. Papsdorf, K. and Brunet, A. (2019) Linking lipid metabolism to chromatin regulation in aging. Trends in cell biology 29 (2), 97-116.

58. Stine, Z.E. et al. (2015) MYC, Metabolism, and Cancer. Cancer Discov 5 (10), 1024-39.

59. Campbell, K.J. and White, R.J. (2014) MYC regulation of cell growth through control of transcription by RNA polymerases I and III. Cold Spring Harbor perspectives in medicine 4 (5), a018408.

60. Conacci-Sorrell, M. et al. (2014) An overview of MYC and its interactome. Cold Spring Harbor perspectives in medicine 4 (1), a014357.

61. Gomez-Roman, N. et al. (2003) Direct activation of RNA polymerase III transcription by c-Myc. Nature 421 (6920), 290.

62. Filer, D. et al. (2017) RNA polymerase III limits longevity downstream of TORC1. Nature 552 (7684), 263.

63. Grewal, S.S. (2015) Why should cancer biologists care about tRNAs? tRNA synthesis, mRNA translation and the control of growth. Biochimica et Biophysica Acta (BBA)-Gene Regulatory Mechanisms 1849 (7), 898-907.

64. Weichhart, T. (2018) mTOR as regulator of lifespan, aging, and cellular senescence: a mini-review. Gerontology 64 (2), 127-134.

65. Turi, Z. et al. (2019) Impaired ribosome biogenesis: mechanisms and relevance to cancer and aging. Aging (Albany NY) 11 (8), 2512.

66. Benayoun, B.A. et al. (2019) Remodeling of epigenome and transcriptome landscapes with aging in mice reveals widespread induction of inflammatory responses. Genome Res 29 (4), 697-709.

67. Davie, K. et al. (2018) A single-cell transcriptome atlas of the aging Drosophila brain. Cell 174 (4), 982-998. e20.

68. Dulken, B.W. et al. (2019) Single-cell analysis reveals T cell infiltration in old neurogenic niches. Nature 571 (7764), 205-210. 
69. Palikaras, K. et al. (2015) Coordination of mitophagy and mitochondrial biogenesis during ageing in C. elegans. Nature 521 (7553), 525.

70. Rana, A. et al. (2017) Promoting Drp1-mediated mitochondrial fission in midlife prolongs healthy lifespan of Drosophila melanogaster. Nature communications 8 (1), 448.

71. Drummond, M.J. et al. (2014) Downregulation of E3 ubiquitin ligases and mitophagy-related genes in skeletal muscle of physically inactive, frail older women: a cross-sectional comparison. Journals of Gerontology Series A: Biomedical Sciences and Medical Sciences 69 (8), 1040-1048.

72. López-Otín, C. et al. (2013) The hallmarks of aging. Cell 153 (6), 1194-1217.

73. Tain, L.S. et al. (2017) A proteomic atlas of insulin signalling reveals tissue-specific mechanisms of longevity assurance. Mol Syst Biol 13 (9), 939.

74. Lawrence, P.A. and Struhl, G. (1996) Morphogens, compartments, and pattern: lessons from drosophila? Cell 85 (7), 951-961.

75. Levine, M. and Davidson, E.H. (2005) Gene regulatory networks for development. Proc Natl Acad Sci U S A 102 (14), 4936-42.

76. Peck, B. et al. (2013) Antagonism between FOXO and MYC regulates cellular powerhouse. Frontiers in oncology $3,96$.

77. Vandenberg, C. et al. (2016) FoxO3 suppresses Myc-driven lymphomagenesis. Cell death \& disease 7 (1), e2046.

78. Tullet, J.M. et al. (2008) Direct inhibition of the longevity-promoting factor SKN-1 by insulin-like signaling in C. elegans. Cell 132 (6), 1025-38.

79. Tepper, R.G. et al. (2013) PQM-1 complements DAF-16 as a key transcriptional regulator of DAF2-mediated development and longevity. Cell 154 (3), 676-690.

80. Dobson, A.J. et al. (2018) Tissue-specific transcriptome profiling of Drosophila reveals roles for GATA transcription factors in longevity by dietary restriction. npj Aging and Mechanisms of Disease 4 (1), 5.

81. Libina, N. et al. (2003) Tissue-specific activities of C. elegans DAF-16 in the regulation of lifespan. Cell $115(4), 489-502$.

82. Kaletsky, R. et al. (2016) The C. elegans adult neuronal IIS/FOXO transcriptome reveals adult phenotype regulators. Nature 529 (7584), 92-6.

83. Greer, C. et al. (2013) Myc-dependent genome instability and lifespan in Drosophila. PloS one 8 (9), e74641.

84. Akagi, K. et al. (2018) Dietary restriction improves intestinal cellular fitness to enhance gut barrier function and lifespan in D. melanogaster. PLoS genetics 14 (11), e1007777.

85. Cai, Y. and Wei, Y.H. (2016) Stress resistance and lifespan are increased in C. elegans but decreased in S. cerevisiae by mafr-1/maf1 deletion. Oncotarget 7 (10), 10812-26.

86. Bonhoure, N. et al. (2015) Loss of the RNA polymerase III repressor MAF1 confers obesity resistance. Genes \& development 29 (9), 934-947.

87. Willis, I.M. (2018) Maf1 phenotypes and cell physiology. Biochimica et Biophysica Acta (BBA)-Gene Regulatory Mechanisms 1861 (4), 330-337.

88. Johnson, D.W. et al. (2014) The Caenorhabditis elegans Myc-Mondo/Mad complexes integrate diverse longevity signals. PLoS genetics 10 (4), e1004278.

89. Castillo-Quan, J.I. et al. (2019) A triple drug combination targeting components of the nutrientsensing network maximizes longevity. Proceedings of the National Academy of Sciences 116 (42), 20817-20819.

90. Hansen, M. et al. (2005) New genes tied to endocrine, metabolic, and dietary regulation of lifespan from a Caenorhabditis elegans genomic RNAi screen. PLoS genetics 1 (1), e17.

91. Hamilton, B. et al. (2005) A systematic RNAi screen for longevity genes in C. elegans. Genes Dev 19 (13), 1544-55.

92. Maklakov, A.A. and Chapman, T. (2019) Evolution of ageing as a tangle of trade-offs: energy versus function. Proc Biol Sci 286 (1911), 20191604. 
93. Partridge, L. and Gems, D. (2002) Mechanisms of aging: public or private? Nature Reviews Genetics 3 (3), 165.

94. Postnikoff, S.D. et al. (2012) The yeast forkhead transcription factors fkh1 and fkh2 regulate lifespan and stress response together with the anaphase-promoting complex. PLoS genetics 8 (3), e1002583.

95. Webb, A.E. et al. (2016) Characterization of the direct targets of FOXO transcription factors throughout evolution. Aging Cell 15 (4), 673-685.

96. Jolma, A. et al. (2013) DNA-binding specificities of human transcription factors. Cell 152 (1-2), 327339.

97. Nitta, K.R. et al. (2015) Conservation of transcription factor binding specificities across 600 million years of bilateria evolution. Elife 4, e04837.

98. Medawar, P.B. (1946) Old age and natural death. Mod. Quart. 2, 30-49.

99. Gil, J. (2019) Cellular senescence causes ageing. Nat Rev Mol Cell Biol 20 (7), 388.

100. Neirijnck, Y. et al. (2019) The Insulin/IGF System in Mammalian Sexual Development and Reproduction. International journal of molecular sciences 20 (18), 4440.

101. Henderson, S.T. and Johnson, T.E. (2001) daf-16 integrates developmental and environmental inputs to mediate aging in the nematode Caenorhabditis elegans. Current Biology 11 (24), 1975-1980. 102. Lee, R.Y. et al. (2001) Regulation of C. elegans DAF-16 and its human ortholog FKHRL1 by the daf2 insulin-like signaling pathway. Current Biology 11 (24), 1950-1957.

103. Shimokawa, I. et al. (2015) The life-extending effect of dietary restriction requires $\mathrm{F}$ oxo3 in mice. Aging cell 14 (4), 707-709.

104. Kuningas, M. et al. (2007) Haplotypes in the human Foxo1a and Foxo3a genes; impact on disease and mortality at old age. European Journal of Human Genetics 15 (3), 294.

105. Timmers, P.R. et al. (2019) Genomics of 1 million parent lifespans implicates novel pathways and common diseases and distinguishes survival chances. Elife 8.

106. Mann, F.G. et al. (2016) Deactivation of the GATA transcription factor ELT-2 is a major driver of normal aging in C. elegans. PLoS genetics 12 (4), e1005956.

107. Budovskaya, Y.V. et al. (2008) An elt-3/elt-5/elt-6 GATA transcription circuit guides aging in C. elegans. Cell 134 (2), 291-303.

108. Tonsaker, T. et al. (2012) Re-evaluating the role of ELT-3 in a GATA transcription factor circuit proposed to guide aging in C. elegans. Mechanisms of ageing and development 133 (1), 50-53.

109. Tullet, J.M. et al. (2017) The SKN-1/Nrf2 transcription factor can protect against oxidative stress and increase lifespan in C. elegans by distinct mechanisms. Aging cell 16 (5), 1191-1194.

110. Lewis, K.N. et al. (2015) Regulation of Nrf2 signaling and longevity in naturally long-lived rodents. Proceedings of the National Academy of Sciences 112 (12), 3722-3727.

111. Mair, W. et al. (2011) Lifespan extension induced by AMPK and calcineurin is mediated by CRTC1 and CREB. Nature 470 (7334), 404.

112. Riera, C.E. et al. (2014) TRPV1 pain receptors regulate longevity and metabolism by neuropeptide signaling. Cell 157 (5), 1023-1036.

113. Arum, O. and Johnson, T.E. (2007) Reduced expression of the Caenorhabditis elegans p53 ortholog cep-1 results in increased longevity. The Journals of Gerontology Series A: Biological Sciences and Medical Sciences 62 (9), 951-959.

114. Ventura, N. et al. (2009) p53/CEP-1 increases or decreases lifespan, depending on level of mitochondrial bioenergetic stress. Aging cell 8 (4), 380-393.

115. Bauer, J.H. et al. (2005) Neuronal expression of p53 dominant-negative proteins in adult Drosophila melanogaster extends life span. Current biology 15 (22), 2063-2068.

116. Zhao, Y. et al. (2018) A polymorphism in the tumor suppressor p53 affects aging and longevity in mouse models. Elife 7, e34701.

117. Maier, B. et al. (2004) Modulation of mammalian life span by the short isoform of p53. Genes \& development 18 (3), 306-319. 
118. Tyner, S.D. et al. (2002) p53 mutant mice that display early ageing-associated phenotypes. Nature 415 (6867), 45.

119. van Heemst, D. et al. (2005) Variation in the human TP53 gene affects old age survival and cancer mortality. Experimental gerontology 40 (1-2), 11-15.

120. Bojesen, S.E. and Nordestgaard, B.G. (2008) The common germline Arg72Pro polymorphism of p53 and increased longevity in humans. cell cycle 7 (2), 158-163. 


\section{Highlights}

Gene expression can be optimised for health and longevity through manipulation of transcription factor (TF) activity. The effects of many such TFs are conserved between animal species indicating evolutionary conservation of underlying mechanisms.

Lifespan-determining TFs regulate a plethora of cellular and organismal functions, including stress resistance, metabolism and growth. They interact with each other both within and between cells. Manipulating their activity in a single cell type can often be sufficient to insure longevity

The evolutionary conservation of their effects on ageing is most likely a reflection of the conservation of their function in processes, such as reproduction, growth and metabolism, that are important earlier in life. Their effects on early and late life can, however, often be uncoupled. 


\section{Outstanding questions}

Is there a minimal set of processes regulated by these TFs that ensures longevity?

Could the complex interactions between TFs and their tissue-specificity help us to decipher their role in lifespan?

How many distinct, longevity-assuring transcriptional programmes can be identified in an animal?

Do these TFs act to counter age-related transcriptional changes?

How well-conserved are the longevity roles of TFs along evolution; can the knowledge gained in simple animal models like Drosophila and $C$. elegans be extended to humans? 